\title{
Camera Calibration and Performance Evaluation of Depth From Defocus (DFD)
}

\author{
Tao Xian, Murali Subbarao \\ Dept. of Electrical \& Computer Engineering* \\ State Univ. of New York at Stony Brook, Stony Brook, NY, USA 11794-2350
}

\begin{abstract}
Real-time and accurate autofocusing of stationary and moving objects is an important problem in modern digital cameras. Depth From Defocus (DFD) is a technique for autofocusing that needs only two or three images recorded with different camera parameters. In practice, there exist many factors that affect the performance of DFD algorithms, such as nonlinear sensor response, lens vignetting, and magnification variation. In this paper, we present calibration methods and algorithms for these three factors. Their correctness and effects on the performance of DFD have been investigated with experiments.
\end{abstract}

Keywords: Autofocusing, Depth From Defocus (DFD), camera calibration, nonlinear sensor response, lens vignetting, magnification variation

\section{INTRODUCTION}

Depth From Defocus (DFD) is a technique for autofocusing that needs only two or three images recorded with different camera parameters. It recovers depth information by estimating the degree of blur. Due to the inherent advantage of being local in nature, the spatial domain approach for DFD is more suitable for real-time autofocusing applications.

In practice, there exist many factors that affect the performance of DFD algorithms. In particular, nonlinear sensor response, lens vignetting, and magnification variation affect the accuracy of DFD. In order to implement DFD on offthe-shelf commercial digital cameras, these factors need to be calibrated and corrected. In this paper, we present new calibration methods for these three factors. Their correctness and effects on performance of DFD have been evaluated with experiments.

Most digital cameras utilize the nonlinear sensor response to extend the dynamic gray-level range through a log-like or gamma transform. DFD theory requires inverse mapping of this non-linear response to linear response through calibration. The intensity measured by the image sensor depends on illumination, exposure period, and reflectance. A method is proposed and tested for correcting this non-linear sensor response.

Optical vignetting is the phenomenon where the effective light energy transmitted by the optical system decreases with increasing inclination of light rays with respect to the optical axis. A vignetting calibration method is implemented and tested for its effects on DFD performance.

In DFD based autofocusing where the lens position is moved, the magnification of an object will change when two images are recorded with different camera parameters. A magnification calibration method is implemented and the estimation error has been evaluated.

The calibration methods for nonlinear response and vignetting correction are direct methods based on illumination measurement using a digital lux tester. They do not need expensive and strictly controlled laboratory environment and

*E-mail: \{txian, murali\}@ece.sunysb.edu; Tel: 1631 632-9149; WWW: www.ece.sunysb.edu/ cvl 
can be used for off-the-shelf cameras. Therefore, these calibration methods should be of general value to other image based algorithms.

\section{STM-DFD ALGORITHM}

The principle of Spatial-domain Convolution/Deconvolution Transform (S Transform) based Depth from Defocus algorithm is proposed by Subbarao and Surya ${ }^{[1]}$, the performance evaluation for different STM based techniques is analysized in Xian and Subbarao ${ }^{[2]}$. Here a brief summary of STM1 is included for further discussion.

According to $\mathrm{S}$ Transform, the focused image $f(x, y)$ can be obtained from its corresponding blur version $g(x, y)$ by local decomposition in the spatial domain.

$$
f(x, y)=g(x, y)-\frac{\sigma_{h}^{2}}{4} \nabla^{2} g(x, y)
$$

where $\sigma_{h}$ is a spread parameter of the point spread function (PSF) $h(x, y)$, And $\nabla^{2}=\frac{\partial^{2}}{\partial x^{2}}+\frac{\partial^{2}}{\partial y^{2}}$ denotes Laplacian operator. For simplicity, the focused image $f(x, y)$ and defocused images $g(x, y)$ are denoted by $f$ and $g$ in the following discussion. If two images $g_{1}$ and $g_{2}$ are recorded with two different parameter settings $\overrightarrow{e_{1}}=\left(s_{1}, f_{1}, D_{1}\right)$ and $\overrightarrow{e_{2}}=\left(s_{2}, f_{2}, D_{2}\right)$, from Eqn. (1) we have

$$
g_{1}-g_{2}=\frac{1}{4} G \nabla^{2} g
$$

where

$$
G=\sigma_{1}^{2}-\sigma_{2}^{2}=\frac{4\left(g_{1}-g_{2}\right)}{\nabla^{2} g}
$$

Under the local third order polynomial model of image brightness, $\nabla^{2} g_{1}=\nabla^{2} g_{2}$. Therefore $\nabla^{2} g_{1}$ and $\nabla^{2} g_{2}$ can be replaced by: $\nabla^{2} g=\left(\nabla^{2} g_{1}+\nabla^{2} g_{2}\right) / 2$

If the lens position is changed during the acquisition of the two images $g_{1}$ and $g_{2}$, the sigma can be calculated from:

$$
\sigma_{2}=\frac{G}{2 \beta}-\frac{\beta}{2}
$$

where $\beta$ is a system parameter. For a specific imaging system, $\beta$ is fixed and can be determined from optical configuration. The focus lens step can be calculated from a Sigma-Lens Step lookup table. ${ }^{[1,2]}$

A new binary mask is introduced to improve the robustness of STM algorithm in [2]. The binary mask is formed by thresholding Laplacian values, which removes unreliable points with low Signal-to-Noise Ratio (SNR). In the Binary Mask based STM1 version of Without Square Without Integration (BM_OSOI), the average of $G$ is calculated based on the binary mask:

$$
G=\frac{4}{U} \sum \sum_{(x, y) \in W} M_{0}(x, y) \frac{\left[g_{1}(x, y)-g_{2}(x, y)\right]}{\nabla^{2} g(x, y)}
$$

where $U=\sum \sum_{(x, y) \in W} M_{0}(x, y)$ is the total weight of the binary mask.

In practice, there exist many factors such as the nonlinear sensor response, lens vignetting, and magnification variation, which may affect the performance of the DFD algorithm. To further understand their effects, the following calibration methods are presented. 


\section{NONLINEAR SENSOR RESPONSE COMPENSATION}

The formation of a digital image on the image sensor of a camera can be described by:

$$
g(x, y)=\int_{0}^{\tau} \int_{0}^{+\infty} q_{s}(x, y, \lambda, t) s(\lambda) d \lambda d t
$$

where $g(x, y)$ is the photo-quantity of the specific sensor element $(x, y) ; q_{s}(x, y, \lambda, t)$ is the actual light energy falling on the image sensor $(x, y) ; s(\lambda)$ is the spectral sensitivity of an element of the sensor. $\tau$ denotes the integration period, which is controlled by exposure time of the camera. From this equation, photo-quantity $g(x, y)$ is neither radiometric nor photometric unit, since it also related to the sensor spectral sensitivity $s(\lambda)$. For a specific camera system, the photo-quality depends on the light energy falling on the sensor cell per unit time, and camera exposure time.

Once the parameters of DFD $\left(s_{1}, s_{2}, f, D\right.$ for STM1) are fixed, the measurement from DFD algorithms should only be related to the object distance, and should not be affected by other changes such as illumination and camera exposure. However, most digital cameras utilize the nonlinear sensor response to extend the dynamic gray-level range through transforms $($ e.g. $\log (\mathrm{z}))$. That means more graylevels are assigned to the photo-quality range with higher probabilities while less graylevels are assigned to the photo-quality range with lower probabilities.

\subsection{Error analysis of non-linear sensor response}

The nonlinear sensor response is a point-wise mapping, which can be formulated by a function $\mathrm{K}$ :

$$
g^{\prime}(x, y)=K[g(x, y)]
$$

where $g^{\prime}(x, y)$ is the distorted intensity after point-wise sensor response mapping, and $g(x, y)$ is the original photoquantity formed as in Eqn. (6).

If digital images are quantized to $\mathrm{n}$ bits, the point-wise sensor mapping can be expressed by the transform vector $\mathbf{k}$ without sacrificing generality:

$$
K(i)=k_{i} I+b
$$

where $b$ is the dark offset, $\mathrm{I}$ is the original photo-quantity vector, and $k_{i}$ is the $i$ th coefficient to map from level $i$ in the original photo-quantity $g$ to distorted intensity $g^{\prime}$.

$$
\begin{aligned}
I & =\left[\begin{array}{llll}
0 & 1 & \Lambda & 2^{n}-1
\end{array}\right]^{\dagger} \\
k & =\left[\begin{array}{llll}
k_{0} & k_{1} & \Lambda & k_{2^{n}-1}
\end{array}\right]
\end{aligned}
$$

For a linear mapping, the components in the coefficient vector for each level should be the same, i.e. $k_{i}=k_{j}=k$; while for a nonlinear mapping, $k_{i} \neq k_{j}$ is valid for some level $i, j$.

Due to nonlinear sensor response, the sigma $\sigma_{2}{ }^{\prime}$ in Eqn. (4) can be calculated from:

$$
\sigma_{2}^{\prime}=\frac{2\left(g_{1}{ }^{\prime}-g_{2}{ }^{\prime}\right)}{\beta \nabla^{2} g^{\prime}}-\frac{\beta}{2}=\frac{4\left(k_{s 1} g_{1}-k_{s 2} g_{2}\right)}{\left(k_{s 1}+k_{s 2}\right) \beta \nabla^{2} g}-\frac{\beta}{2}
$$

where $g_{1}{ }^{\prime}$ and $g_{2}{ }^{\prime}$ correspond to the distorted blur images acquired at different lens step.

The sigma error due to sensor response $\varepsilon$ is expressed by the difference between sigma calculated from distorted image pairs and the ones without distortion.

$$
\varepsilon=\sigma_{2}{ }^{\prime}-\sigma_{2}=\frac{k_{s 1}-k_{s 2}}{k_{s 1}+k_{s 2}} \frac{2\left(g_{1}+g_{2}\right)}{\beta \nabla^{2} g}
$$

From Eqn. (12), the sigma error $\varepsilon$ is not only related to the system parameter $\beta$, but also depends on camera nonlinear mapping coefficient and the image itself. The sigma error $\varepsilon$ is transferred to the step error through the sigma-step mapping $S S(\sigma, \varepsilon)$. A dependence on illumination and/or exposure is hence introduced. This is not desirable in autofocusing applications. 
This sigma error $\varepsilon$ can be eliminated only if $k_{s 1}=k_{s 2}$ in Eqn. (12). Since the value of $g_{1}$ and $g_{2}$ are arbitrary intensities in the range of $\left[0,2^{n}-1\right]$, the following equation should be valid for any intensity to compensate for $\varepsilon$ :

$$
k_{s 1}=k_{s 2}=k
$$

Eqn. (13) demonstrates $\varepsilon$ can be eliminated when there exists a linear relation between observed intensity and photoquantity. The linearization can be obtained by inverse mapping of sensor response $K^{-1}$.

$$
g_{0}=K^{-1}\left(g^{\prime}\right)=K^{-1}[K(g)]=g
$$

\subsection{Direct calibration}

Sensor response calibration is needed to compensate for its non-linearity. There are several approaches to measure the sensor response, through statistical averaging on the whole image for arbitrary scenes, such as [3] and [4]. However averaging on the image plane includes the effect of vignetting, which will be discussed in Section 4 . The resulting response is a weighted average of on-axis points and off-axis points, and the weight depends on scene content.

A direct measurement method is presented here to calibrate the nonlinear response for off-the-shelf cameras. The setup of the nonlinear sensor response calibration is briefly outlined in Fig. 1. A diffusive white screen WS is illuminated by multiple light sources from $L_{1}$ to $L_{4}$. The light sources are controlled from the linear lamp controller module $L C$ to create variable/adjustable illumination. The intensity at the central area of the white screen is measured by a Digital Lux Tester YF-1065. The image of the white screen is acquired by a digital camera to be calibrated. A lookup table is established by changing the illumination incrementally while recording the image at each illumination step. The relationship between camera gray-levels and normalized illumination gray-levels is shown in Fig. 2. Mean brightness in a $10 * 10$ image region is used for reducing noise in the central area of the white screen.

To evaluate the effect of nonlinear sensor response, a series of DFD experiments were conducted under different photoquantity conditions. The ambient illumination is 253 lux measured at the center of an object plane, and the distance from the front surface of lens to the object plane is $540 \mathrm{~mm}$. According to Eqn. (6), the photo-quantity can be changed by either illumination level or camera shutter speed. We control the shutter speed to obtain a wider range on photoquantity. The shutter speed changes from $15.625 \mathrm{~ms}$ to $500 \mathrm{~ms}$, which correspond to a change factor of 32 in photoquantity, as displayed in Fig. 3. In Fig.3, Images range from under-exposed as in (a), (b), to over-exposed as in (e), (f) . The photo-quantity is doubled at each stage from (a) to (f), however the gray level of observed image does not increase correspondingly due to camera range compression. About 384 DFD experiments were conducted at 8 different random positions and 6 different exposure levels. The 8 randomly selected positions are 325.1, 383.1, 474.1, 538.6, 630.0, $784.2,1058.4$ and $1353.6 \mathrm{~mm}$ respectively, which are measured from the front surface of the lens. The corresponding lens steps are obtained from Depth From Focus (DFF) experiments, and they are 25, 38, 58, 74, 101, 122, 131 and 145 lens step respectively.

As shown in Fig. 4, STM1 without sensor response compensation has a mean error of up to 14 lens steps (Distance $630.0 \mathrm{~mm}$, Shutter Speed $15.625 \mathrm{~ms}$ ), while the corresponding RMS lens step error is 0.354 step, the mean error reflects a systematic bias which is predicted by Eqn. (12). After nonlinear sensor response compensation, at the same photoquantity, the mean lens step error is reduced to 1.667 step, and all 8 DFD measurements get exactly the same step number, since RMS lens step error is 0 . The detailed results of DFD without/with nonlinear sensor response compensation are summarized in Table 1 and 2. Table 1 shows the mean and RMS lens step error before nonlinear sensor response compensation, while Table 2 shows errors after sensor response calibration.

When the photo-quantity continues to increase from (e) to (f), step shifts in far field for both DFF and DFD can be observed. In the image (f), the sensor is already saturated, and the observed image is no longer a correct measure of photo-quantity. In this extreme condition, the error of DFD with sensor compensation $(-4.431 \pm 0.236$ step $)$ is still better than the one without compensation $(-8.667 \pm 0.535$ step $)$. 


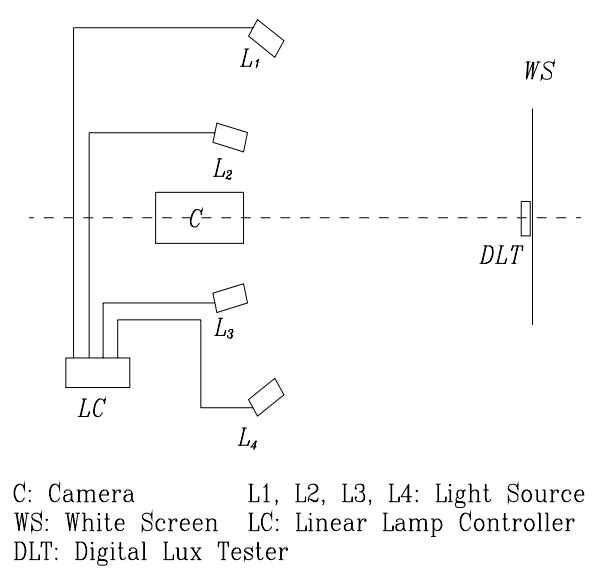

Figure 1. Setup for nonlinear sensor response calibration

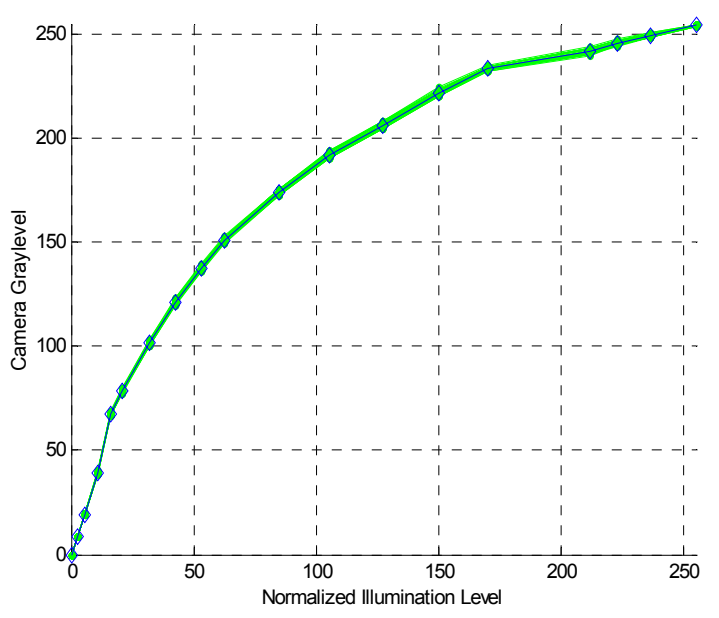

Figure 2. Nonlinear sensor response

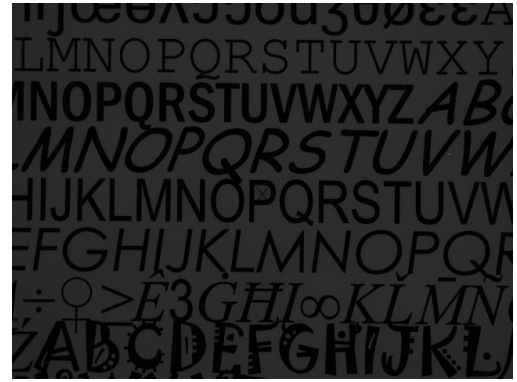

(a) $1 \mathrm{x}$

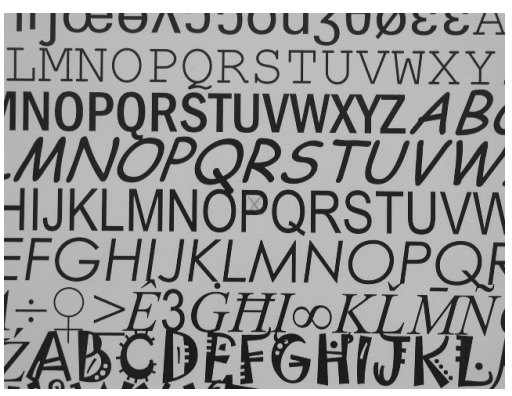

(d) $8 \mathrm{x}$

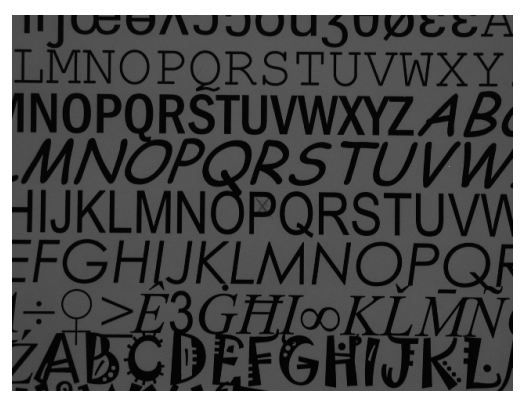

(b) $2 \mathrm{x}$

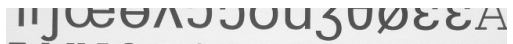
IMNOPORSTUVWXY INOPQRSTUVWXYZ $A \bar{B}_{\text {, }}$ MNOPQRSTUVW -IJKLMNOPQRSTUVM EFGHIJKLMNOPQF $\div \div B 3 G H L O K L M N$ ABCDEGHPK:

(e) $16 \mathrm{x}$

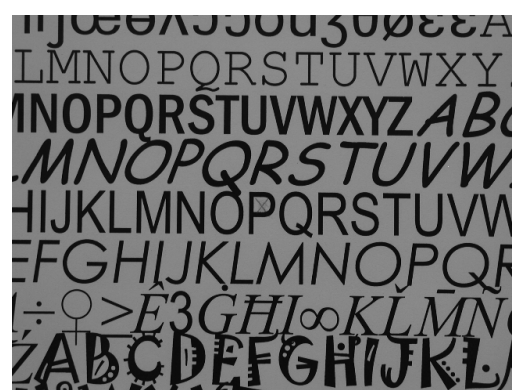

(c) $4 \mathrm{x}$

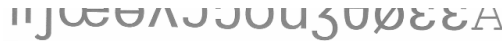
IMNOPORSTUVWXY INOPQRSTUWWXYZAB MNOPQRSTUVM IIJKLMNOPQRSTUVM =FGHLJKLMNOPQF $\div \bigcirc>\mathrm{F}_{3}$ GIILKL $\overline{M N}_{1}$ YABC DEG

(f) $32 x$

Figure 3. Images obtained at different photo-quantity

From (a) to (f), images are captured with exposure time of 15.625, 31.25, 62.5, 125, 250 and $500 \mathrm{~ms}$. Assume the photo-quantity in (a) as a unit measure, the photo-quantity doubles in each stage from (a) to (f) 


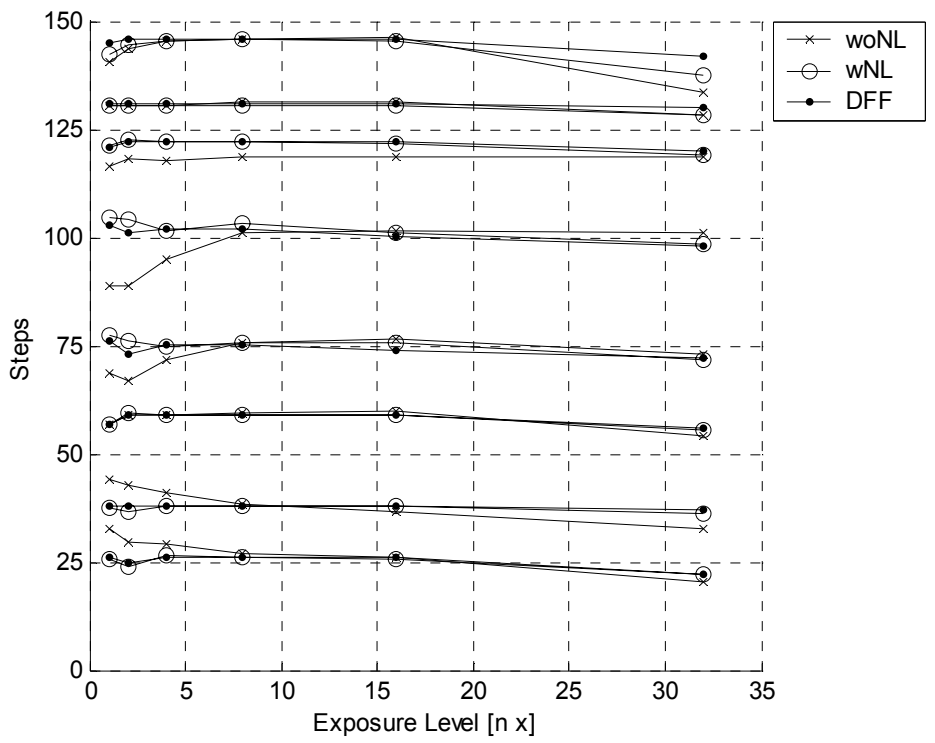

Figure 4. DFD Results without/with nonlinear sensor response calibration woNL: DFD STM1 without Non-Linear sensor response compensation wNL: DFD STM1 with Non-Linear sensor response compensation DFF: Depth From Focus

\begin{tabular}{l|cc|cc|cc|cc|cc|cc|}
\hline & \multicolumn{2}{|c|}{ Exposure 1 } & \multicolumn{2}{c|}{ Exposure 2 } & \multicolumn{2}{c|}{ Exposure 3 } & \multicolumn{2}{c|}{ Exposure 4 } & \multicolumn{2}{c}{ Exposure 5 } & \multicolumn{2}{c}{ Exposure 6 } \\
& Mean & Std & Mean & Std & Mean & Std & Mean & Std & Mean & Std & Mean & Std \\
\hline Pos 1 & 6.833 & 0.000 & 4.583 & 0.463 & 3.083 & 0.463 & 0.833 & 0.000 & -0.042 & 0.354 & -1.542 & 0.518 \\
Pos 2 & 6.167 & 0.000 & 4.917 & 0.463 & 3.167 & 0.000 & 0.167 & 0.000 & -1.333 & 0.535 & -4.208 & 0.518 \\
Pos 3 & -0.417 & 0.463 & -0.167 & 0.000 & -0.167 & 0.000 & 0.333 & 0.535 & 0.708 & 0.354 & -2.042 & 0.354 \\
Pos 4 & -7.167 & 0.000 & -6.042 & 0.354 & -3.167 & 0.000 & 0.458 & 0.518 & 2.333 & 0.535 & 1.208 & 0.518 \\
Pos 5 & -14.125 & 0.354 & -12.250 & 0.707 & -6.875 & 0.641 & -0.750 & 0.463 & 1.750 & 0.463 & 3.250 & 0.707 \\
Pos 6 & -4.625 & 0.991 & -3.750 & 0.463 & -4.125 & 0.518 & -3.250 & 0.463 & -3.250 & 0.463 & -1.500 & 0.535 \\
Pos 7 & -0.583 & 0.463 & -0.583 & 0.463 & -0.708 & 0.354 & 0.292 & 0.354 & 0.417 & 0.463 & -1.833 & 0.000 \\
Pos 8 & -4.292 & 0.835 & -2.292 & 0.991 & -0.542 & 1.061 & -0.042 & 0.991 & 0.458 & 0.744 & -8.667 & 0.535 \\
\hline
\end{tabular}

Table 1. DFD lens step error by mean and rms without nonlinear sensor compensation

\begin{tabular}{l|cc|cc|cc|cc|cc|cc}
\hline & \multicolumn{2}{|c|}{ Exposure 1 } & \multicolumn{2}{c|}{ Exposure 2 } & \multicolumn{2}{c|}{ Exposure 3 } & \multicolumn{2}{c|}{ Exposure 4 } & \multicolumn{2}{c}{ Exposure 5 } & \multicolumn{2}{c}{ Exposure 6 } \\
& Mean & Std & Mean & Std & Mean & Std & Mean & Std & Mean & Std & Mean & Std \\
\hline Pos 1 & -0.347 & 0.236 & -1.139 & 0.309 & 0.611 & 0.000 & -0.056 & 0.000 & -0.347 & 0.236 & 0.278 & 0.000 \\
Pos 2 & -0.528 & 0.309 & -1.278 & 0.000 & 0.056 & 0.000 & -0.153 & 0.345 & -0.278 & 0.000 & -0.861 & 0.309 \\
Pos 3 & -0.139 & 0.309 & 0.236 & 0.236 & -0.056 & 0.000 & -0.056 & 0.000 & 0.111 & 0.356 & -0.639 & 0.309 \\
Pos 4 & 1.194 & 0.309 & 2.986 & 0.236 & -0.056 & 0.000 & 0.694 & 0.309 & 1.528 & 0.309 & -0.181 & 0.345 \\
Pos 5 & 1.667 & 0.000 & 3.292 & 0.236 & -0.500 & 0.356 & 1.250 & 0.309 & 1.250 & 0.309 & 0.292 & 0.427 \\
Pos 6 & 0.083 & 0.309 & 0.375 & 0.345 & 0.083 & 0.309 & 0.167 & 0.000 & -0.125 & 0.236 & -0.958 & 0.345 \\
Pos 7 & -0.611 & 0.000 & -0.569 & 0.236 & -0.611 & 0.000 & -0.319 & 0.236 & -0.611 & 0.000 & -1.611 & 0.000 \\
Pos 8 & -2.472 & 0.992 & -1.306 & 0.690 & -0.514 & 0.496 & -0.347 & 0.661 & -0.556 & 0.356 & -4.431 & 0.236 \\
\hline
\end{tabular}

Table 2. DFD lens step error by mean and rms with nonlinear sensor compensation 


\section{LENS VIGNETTING COMPENSATION}

Optical vignetting is the phenomenon wherein the effective light energy transmitted by the optical system decreases with increasing inclination of light rays with respect to the optical axis.

The consequence of optical vignetting for a focused scene is merely a reduced brightness towards the image corners. However, optical vignetting can also have a pronounced effect on out-of-focus parts of the image. Because the shape of an Out-Of-Focus Highlight (OOFH) mimics the shape of the clear aperture, this leads to the so-called cat's eye effect. With an increasing distance from the optical axis the shape of the OOFH progressively narrows and starts to resemble a cat's eye. The larger the distance from the image center, the narrower the cat's eye becomes.

A vignetting calibration method is used to evaluate the effect of vignetting on DFD measurement. If a uniform illumination is available, the vignetting coefficient could be simply calculated from a single image of a diffusive white screen. However it is difficult to obtain a uniform illumination that is accurate, although not impossible. An alternative way is used in our calibration. The setup for vignetting calibration was similar to that in Fig.1. A $5 * 5$ grid pattern is used as a calibration pattern (see Fig. 3(a) ). In each grid, illumination is measured by the Digital Lux Tester YF-1065 at the center of grids, and the image of the grid pattern is captured by the camera. The gray level obtained is a transformed value of real photo-quantity due to the nonlinearity of sensor response. A lookup table for the reverse mapping discussed in Section 3 is used. The vignetting coefficient is calculated by the ratio of illumination intensity at pixel $(x, y)$ to the intensity at the center of the image. Due to the rotational symmetry property, the relation between vignetting coefficient and pixel distance in polar coordinate is obtained from a third-order polynomial fitting:

$$
V(\rho)=-3.1064 * 10^{-9} * \rho^{3}+3.2488 * 10^{-7} * \rho^{2}-6.7845 * 10^{-5} * \rho+1
$$

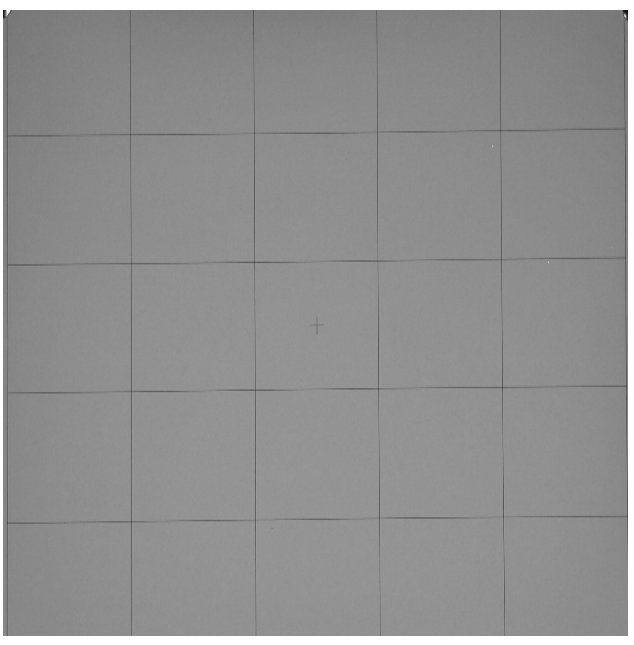

(a)

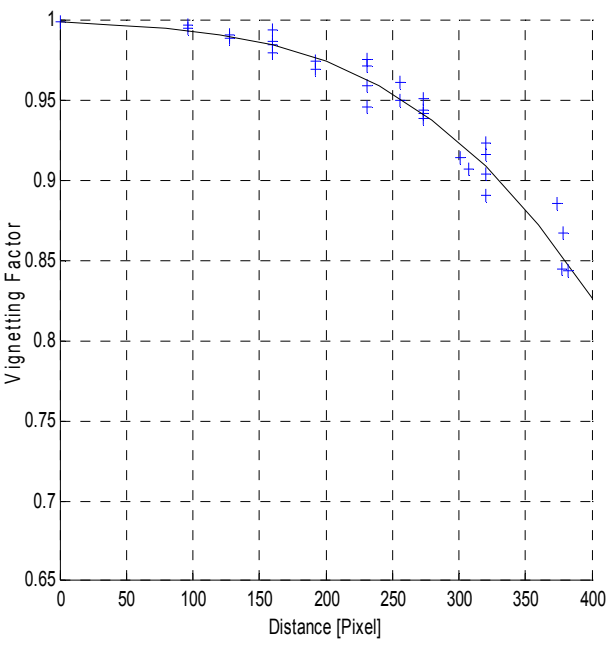

(b)

Figure 5. Vignetting calibration

(a) Pattern (b) Result

The result of vignetting factor vs. pixel distance is shown in Fig. 5(b). From Fig. 5(b), if the DFD AF window is in the center area, the distortion of vignetting can be ignored. (for a $96^{*} 96$ focusing window, the intensity attenuation is $0.24 \%$ ). When the focusing window is near a corner of the view, there could be a $12.1 \%$ difference in the diagonal direction. In this case, vignetting should be compensated by multiplying the reciprocal of the corresponding vignetting coefficient. 


\section{MAGNIFICATION CALIBRATION}

In STM1, when the object to be focused is fixed, there is a magnification change between defocused images acquired at two different lens steps. A magnification calibration method is developed to determine this variation.

A chessboard pattern whose grid size is $15 \mathrm{~mm}$ by $15 \mathrm{~mm}$, is captured by the camera at focus steps 35 and 98 , and the corner points are detected as shown in Fig. 6 . The distance between the camera and the chessboard pattern is $500 \mathrm{~mm}$, which corresponds to the focused image approximately at step 62. For convenience, we define the defocused image at step 35 as image 1, and the one at step 98 as image 2 . The transformation between image 1 and image 2 can be calculated through a projection matrix.

The corners on image 1 and image 2 are detected and sorted row by row into two corner arrays respectively. Corners on the same position of array make a corresponding corner pair. There are $11 * 8$ corner pairs as demonstrated in Fig. $6(\mathrm{a})$ and (b).

For each corresponding corner pair, the coordinates of the corners can be expressed as:

$$
V_{i}=M U_{i}, \quad i \in N
$$

where $U_{i}$ and $V_{i}$ are coordinates of corner pairs in image 1 and image 2 respectively. These coordinates are expressed in projective space, i.e. $U_{i}=\left[\begin{array}{lll}x_{1, i} & y_{1, i} & 1\end{array}\right]^{t}$, and $V_{i}=\left[\begin{array}{lll}x_{2, i} & y_{2, i} & 1\end{array}\right]^{t} . N$ is the total number of corresponding pairs. $M$ is a $3 * 3$ transformation matrix. Since we have no prior knowledge about the transformation, there are 9 unknown elements in $M$.

For $N$ pairs of corner pairs, a least-square matrix can be obtained:

$$
A M^{\prime}=b
$$

where $A$ is a $3 \mathrm{~N}^{*} 9$ matrix, and $b$ is a $3 \mathrm{~N}$ column vector that is made from lapping over $V_{i}, \quad i=1, \Lambda, N$.

$$
\begin{aligned}
A & =\left[\begin{array}{ccccccccc}
x_{1,1} & y_{1,1} & 1 & 0 & 0 & 0 & 0 & 0 & 0 \\
0 & 0 & 0 & x_{1,1} & y_{1,1} & 1 & 0 & 0 & 0 \\
0 & 0 & 0 & 0 & 0 & 0 & x_{1,1} & y_{1,1} & 1 \\
\mathrm{M} & \mathrm{M} & \mathrm{M} & \mathrm{M} & \mathrm{M} & \mathrm{M} & \mathrm{M} & \mathrm{M} & \mathrm{M} \\
x_{1, N} & y_{1, N} & 1 & 0 & 0 & 0 & 0 & 0 & 0 \\
0 & 0 & 0 & x_{1, N} & y_{1, N} & 1 & 0 & 0 & 0 \\
0 & 0 & 0 & 0 & 0 & & x_{1, N} & y_{1, N} & 1
\end{array}\right] \\
b & =\left[\begin{array}{llllllll}
x_{2,1} & y_{2,1} & 1 & \Lambda & x_{2, N} & y_{2, N} & 1
\end{array}\right]^{t}
\end{aligned}
$$

and $M^{\prime}$ is reorganized from $M$ :

$$
M^{\prime}=\left[\begin{array}{lllllllll}
m_{11} & m_{12} & m_{13} & m_{21} & m_{22} & m_{23} & m_{31} & m_{32} & m_{33}
\end{array}\right]^{t}
$$

Then the transform matrix $M$ can be calculated by:

$$
M^{\prime}=\left(A^{t} A\right)^{-1} A^{t} b
$$

The transformation matrix $M$ between image 1 and image 2 is calculated and reformed from Eqn. (21):

$$
M=\left[\begin{array}{ccc}
1.0083 & 0 & -2.6526 \\
0 & 1.0082 & -2.0510 \\
0 & 0 & 1
\end{array}\right]
$$

Using this matrix $M$ to project image 1 to image 2, the projection error for each corner is plotted in Fig. 7, the projection error can be expressed by mean and RMS pixel error $(-2.283 \mathrm{e}-013 \pm 0.061,-1.389 \mathrm{e}-014 \pm 0.050)$, and the maxim error is less than 0.2 pixel both in $\mathrm{x}$ and $\mathrm{y}$ direction. The new image can be generated by a bicubic interpolation of image 1. 


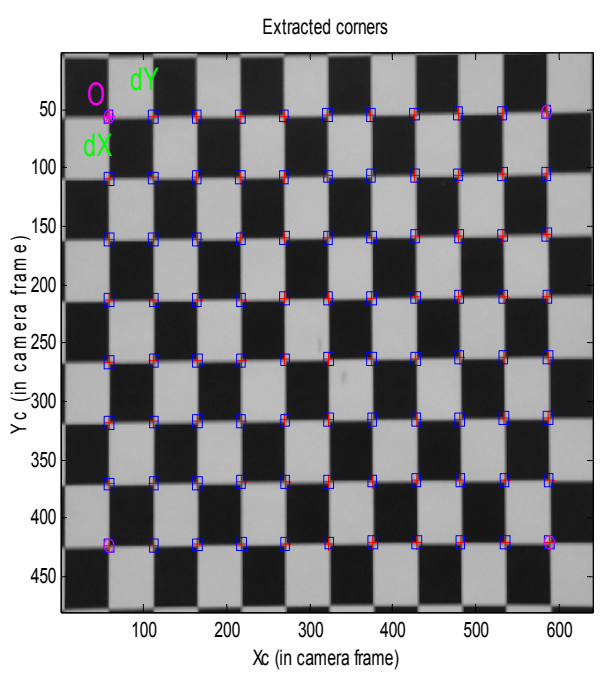

(a)

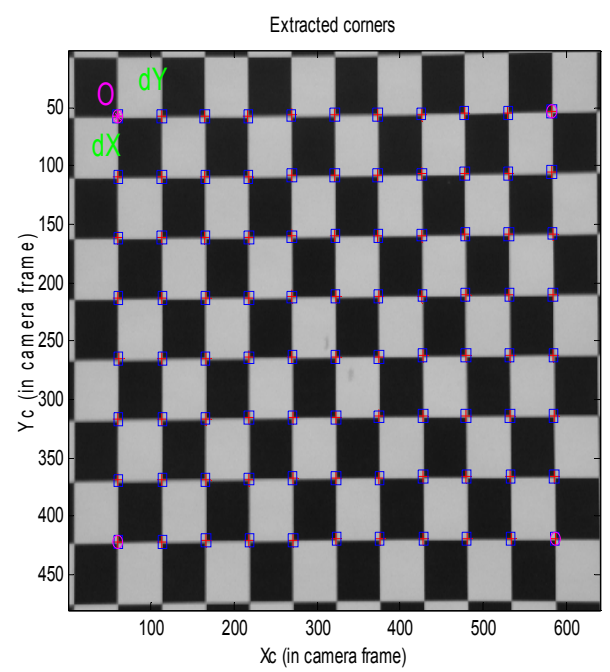

(b)

Figure 6. Magnification calibration using pattern captured at different steps (a) chessboard pattern captured at step 35 (b) chessboard pattern captured at step 98

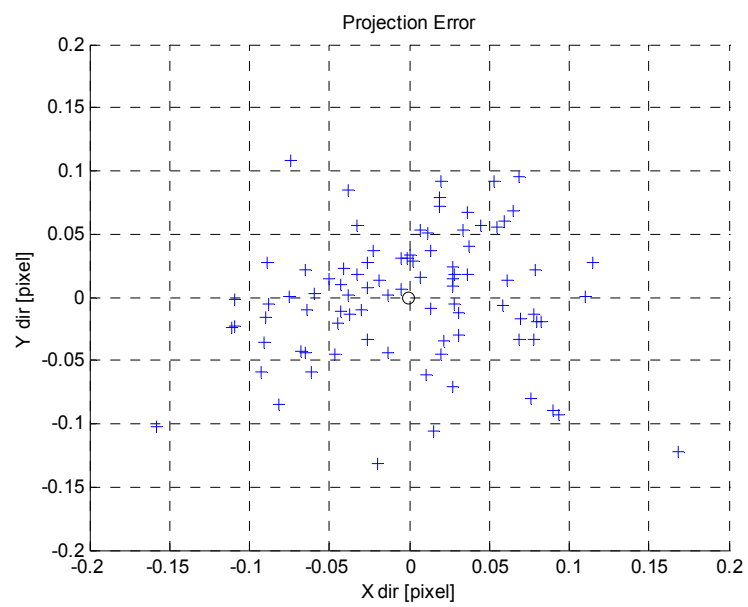

Figure 7. Project error from estimated transformation matrix $M$

\section{CONCLUSION}

In this paper, calibration methods and procedures for nonlinear sensor response, optical vignetting, and magnification variation are presented. The correctness and effects on the performance of DFD have been evaluated with experiments. These calibrations do not need expensive and strictly controlled laboratory environment. They can be used for off-theshelf cameras. Therefore, these calibration methods should be of general value to other image based algorithms. 


\section{REFERENCES}

1. M. Subbarao and G. Surya, "Depth from Defocus: A Spatial Domain Approach", International Journal of Computer Vision, Vol. 13, No. 3, p. 271-294,1994

2. T. Xian, and M. Subbarao, "Performance Evaluation of Different Depth-From-Defocus (DFD) Techniques", Proc. of SPIE, Boston, Oct. 2005

3. S. Mann, "Comparametric Equations with Practical Applications", IEEE Trans. Image Processing, Vol. 9, No. 8, P1389-1406, 2000

4. S.Y. Park, and M. Subbarao, "Automatic Focusing of a Digital Still Camera using a Depth-from Defocus Technique: An Approach for Compensating Non-Linear Camera Response Function”, Internal Technical Report, Computer Vision Lab, ECE, State Univ. of New York at Stony Brook, 2004 